\title{
Use of Pareto optimisation for tuning power system stabilizers
}

\author{
S. PASZEK* \\ Institute of Electrical Engineering and Informatics, Silesian University of Technology, 10 Akademicka St., 44-100 Gliwice, Poland
}

\begin{abstract}
The paper presents a method for determining sets of Pareto optimal solutions (compromise sets) - parameter values of PSS3B system stabilizers working in a multi-machine power system - when optimising different multidimensional criteria. These criteria are determined for concrete disturbances when taking into account transient waveforms of the instantaneous power, angular speed and terminal voltage of generators in one, chosen generating unit or in all units of the system analysed. The application of multi-criteria methods allows taking into account the optimisation process of power system stabilizer (PSS) parameters, many sometimes contradictory requirements (criteria) without losing ability to reach the optimal solution. A choice of the compromise solution can be made by assuming the values of the weighting coefficients associated with particular components of the vector criterion and determining the equivalent, global criterion. A change of the values of those weighting coefficients in the equivalent criterion does not require, in the case of the Pareto optimization, carrying out repeated calculations.
\end{abstract}

Key words: Pareto optimisation, power system stabilizers, power system, electromechanical swings.

\section{Introduction}

Optimisation in Pareto sense (polyoptimisation, vector optimisation, multicriteria optimisation) is a generalization of a classical, single criterion optimisation. It has been applied to solving problems in engineering for which an explicit quality factor does not exist [1-3]. Optimisation consists in improving one quality factor, and the optimal solution is a set of the optimal values of selected parameters [4]. Whereas, when performing the Pareto optimisation one determines a set of Pareto optimal solutions, i.e. compromise set [1-3], being the result of simultaneous searching the extrema of many factors called aspects. The solution of the Pareto optimisation is a set of the optimal values of selected parameters, e.g. parameters of PPSs working in a multi-machine power system (PS).

The compromise set $\boldsymbol{\Lambda}$ is a hypersurface in $r$-dimensional objective space $\boldsymbol{Q}$ [5], where $r$ is a number of the quality factors optimised. The objective space is determined by the achievable values of the quality factors (partial objective functions) $Q_{i}$ assumed for optimisation. Since the aspects $Q_{i}$ are functions of the optimised parameters $x_{j}$, the objective space $\boldsymbol{Q}$ is an image of $l$-dimensional control space $\boldsymbol{X}$ [5], where $l$ is the number of the optimised parameters.

The vector $\widetilde{\boldsymbol{Q}}=\left\{\widetilde{Q}_{1}, \widetilde{Q}_{2}, \ldots, \widetilde{Q}_{r}\right\}$ called uniformly better than the vector $\boldsymbol{Q}=\left\{Q_{1}, Q_{2}, \ldots, Q_{r}\right\}$ belongs to the compromise set $\boldsymbol{\Lambda}$ if for all components of the vectors there is $\widetilde{Q}_{i} \leq Q_{i}$ and there exists at least one component for which $\widetilde{Q}_{i}<Q_{i}$, where the symbol . denotes the optimal value in Pareto optimisation sense ( $\widetilde{Q}_{i}-$ optimal value of the $i$-th criterion, $\widetilde{x}_{j}$ - optimal value of the $j$-th parameter).

The compromise set definition can be also written with the use of a concept of direction of simultaneous improvement. Whereas the direction of simultaneous improvement is called such a change of the optimised parameters $x_{j}$ ap- pears, which causes simultaneous improvement of all assessment criteria [2,3]. Then all such points of the objective space $\widetilde{\boldsymbol{Q}}=\left\{\widetilde{Q}_{1}, \widetilde{Q}_{2}, \ldots, \widetilde{Q}_{r}\right\} \in \boldsymbol{Q}$ dependent on controls $\widetilde{\boldsymbol{X}}=\left\{\widetilde{x}_{1}, \widetilde{x}_{2}, \ldots, \widetilde{x}_{l}\right\}$ for which the direction of simultaneous improvement does not exist, belong to the compromise set $\boldsymbol{\Lambda}[1]$.

The optimal solutions fulfilling the relation

$$
\left\{\widetilde{Q}_{1}, \widetilde{Q}_{2}, \cdots, \widetilde{Q}_{r}\right\} \in \boldsymbol{\Lambda} \Leftrightarrow \neg \exists\left\{Q_{1}, Q_{2}, \cdots, Q_{r}\right\} \in \boldsymbol{Q},
$$

belong to the compromise set $\boldsymbol{\Lambda}$, where

$$
\begin{gathered}
Q_{i} \leq \widetilde{Q}_{i} \text { for each } i \in\langle 1,2, \cdots, r\rangle, \\
Q_{i}<\widetilde{Q}_{i} \text { for at least one } i \in\langle 1,2, \cdots, r\rangle,
\end{gathered}
$$

and the symbol $\neg \exists$ denotes "does not exist".

The exemplary compromise set for two-dimensional objective space is shown in Fig. 1.

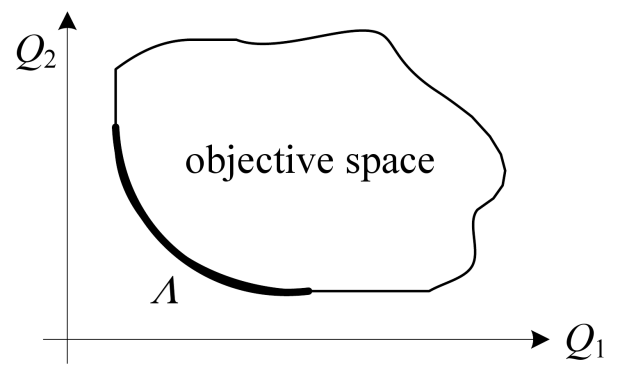

Fig. 1. Exemplary compromise set for two-dimensional objective space

The last stage of the Pareto optimisation is a selection of one solution from among all compromise solutions [5]. The selected solution is the optimal one in classical formulation,

*e-mail: Stefan.Paszek@polsl.pl 
since it can be proved that it is the extremum of one equivalent factor being a weighted sum of the Pareto optimisation aspects when assuming appropriate weighting coefficients $[2,5]$.

\section{Vector quality criterion for tuning power system stabilizers}

Due to complexity of phenomena occurring in the power system, the process of PSS parameter optimisation should take into consideration many criteria associated with damping electromechanical swings as well as limiting voltage changes in particular generating units during different disturbances of the steady state [6]. The objective function being minimised has to contain different components connected with the optimised criteria. One of the basic problems when determining the objective function is appropriate selection of the weighting coefficients corresponding to particular components of the function. Additionally, the analysis is complicated by the fact that in the objective function there are criteria contradictory to each other. Moreover, the assumed values of those weighting coefficients in the additive objective function influence significantly the final results of optimisation.

The solution to that problem can be an application of the Pareto optimisation which enables taking into account different and contradictory criteria simultaneously [2], e.g. minimisation of deviations of the instantaneous power, angular speed and terminal voltage of particular generators working in the PS in different disturbance states [7-10].

In the case of selection of PSS parameters, the result of the Pareto optimisation is a set of optimal solutions, i.e. the compromise set. In the case of the classical optimisation, the solution is one set of the searched parameters of the optimised stabilizers.
For the classical and Pareto optimisation numerical algorithms are used. They enable calculations of a single or vector objective function and its minimisation. There can be distinguished algorithms for searching local extrema (e.g. gradient methods) and global algorithms (e.g. genetic and hybrid algorithms). In the investigations presented there was used a hybrid algorithm being a series connection of genetic and gradient algorithms. The results of the genetic algorithm are the starting point for the gradient algorithm. The genetic algorithm, adapted to optimising many criteria simultaneously, searches the global minimum of the vector objective function in the determined range of the searched parameters. The starting point is randomly selected from the search range, so it is not necessary to determine it accurately. However, that algorithm is slowly convergent. The gradient algorithm is faster convergent, but it searches the local minimum of a single, selected (from the whole vector) objective function. A series connection of the genetic and gradient algorithms eliminates their main drawbacks [2]. Adaptation of the gradient algorithm to multi-criteria optimisation consisted in modification of the selection method. In computations there was used a selection with a tournament method of $r$ simultaneous tournaments [2,11] (where $r$ determines a number of the optimised criteria).

\section{Mathematical model of a multi-machine power system}

A mathematical model of a power system in the MatlabSimulink environment was developed for simulations. First, a general model of the generating unit was constructed (Fig. 2). In this model, using the "Configurable Subsystems" type blocks, it is possible to create a model of the generating unit when choosing the specific model of: a synchronous generator, an excitation system, a turbine and a PSS.

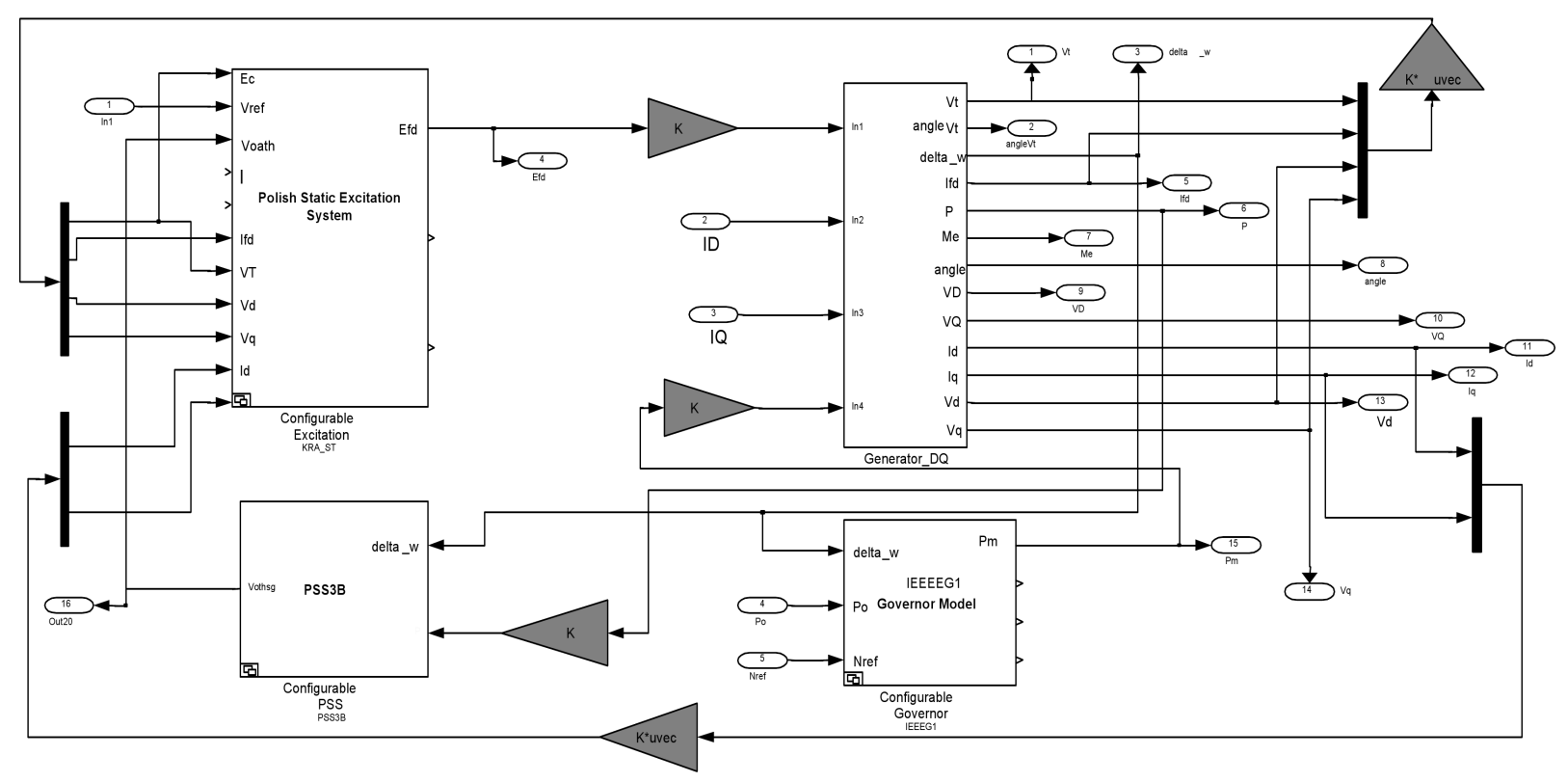

Fig. 2. Structural model of the generating unit 
It was assumed that when investigating relatively slowvariable transient states, the transformation voltages were neglected in the approximate equations of the quasi-stationary state (of electromechanical waveforms) related to the generator stator equivalent circuits, which in consequence brought them to algebraic equations. The above assumption also results in algebraization of the voltage-current equations of the power network, which makes the analysis of the PS operation significantly easier.

The generator state equations expressed in relative network units $[6,12,13]$ can be given in the following form:

$$
\begin{gathered}
\frac{\mathrm{d} \boldsymbol{X}_{\mathrm{M}}}{\mathrm{d} t}=\boldsymbol{M}_{\mathrm{M}} \boldsymbol{X}_{\mathrm{M}}+\boldsymbol{N}_{\mathrm{m}} \boldsymbol{I}_{\mathrm{m}}+\boldsymbol{B}_{\mathrm{MF}} E_{\mathrm{fd}}+\boldsymbol{f}_{\mathrm{sat}}\left(\boldsymbol{X}_{\mathrm{M}}\right), \\
\frac{\mathrm{d} \omega}{\mathrm{d} t}=\frac{1}{T_{\mathrm{m}}}\left(P_{\mathrm{m}} / \omega-M_{\mathrm{e}}\right), \\
\frac{\mathrm{d} \delta}{\mathrm{d} t}=\omega_{\mathrm{N}}(\omega-1), \\
\boldsymbol{U}_{\mathrm{m}}=\boldsymbol{K}_{\mathrm{m}} \boldsymbol{X}_{\mathrm{M}}-\boldsymbol{Z}_{\mathrm{m}} \boldsymbol{I}_{\mathrm{m}},
\end{gathered}
$$

where $\boldsymbol{X}_{\mathrm{M}}-$ generator electromagnetic state variables linearly dependent on the fluxes linked with the rotor circuits, $\boldsymbol{U}_{\mathrm{m}}=\left[V_{\mathrm{d}}, V_{\mathrm{q}}\right]^{\mathrm{T}}, \boldsymbol{I}_{\mathrm{m}}=\left[I_{\mathrm{d}}, I_{\mathrm{q}}\right]^{\mathrm{T}}-$ voltages and currents of the armature in d and q axis, $E_{\mathrm{fd}}, P_{\mathrm{m}}, M_{\mathrm{e}}, \delta, \omega_{\mathrm{N}}, T_{\mathrm{m}}-$ field voltage, turbine mechanical power, electromagnetic torque, power angle, rated angular speed, mechanical time constant, $\boldsymbol{M}_{\mathrm{M}}$, $\boldsymbol{N}_{\mathrm{m}}, \boldsymbol{B}_{\mathrm{MF}}, \boldsymbol{K}_{\mathrm{m}}, \boldsymbol{Z}_{\mathrm{m}}$ - matrices dependent on the electromagnetic parameters of the generator model, $\boldsymbol{f}_{\text {sat }}$ - nonlinear function determining the generator magnetic circuit saturation.

Configurable Excitation, Configurable Governor and Configurable PSS blocks of Fig. 2 represent the state and output equations of the selected excitation system, turbine and PSS models (in relative regulator units) $[6,12,13]$. The other (shaded) blocks in Fig. 2 include appropriate correction coefficients that allow making relations between the quantities expressed in different relative units.

The application of network relative units to the state equations of generators as well as transformation of currents and voltages of generator armatures to the common coordinate system D, Q rotating with the angular speed $\omega_{\mathrm{N}}$, which is assumed to be equal to the average angular speed of all synchronous machines under steady state conditions, enables obtaining the convenient relations between the state equations of the particular generating units and the voltage-current equations of the power network. The relative network quantities are determined when assuming the common reference power (base power) for all generating units equal, for instance, $S_{\text {ref }}=100 \mathrm{MW}$.
There are valid the following relationships between the quantities in the coordinate system $\mathrm{d}, \mathrm{q}$ and those in the system D, Q [6]:

$$
\boldsymbol{W}_{\mathrm{d}, \mathrm{q}}=\boldsymbol{t}_{\mathrm{r}} \boldsymbol{W}_{\mathrm{D}, \mathrm{Q}}, \quad \boldsymbol{W}_{\mathrm{D}, \mathrm{Q}}=\boldsymbol{t}_{\mathrm{r}}^{-1} \boldsymbol{W}_{\mathrm{d}, \mathrm{q}},
$$

where transformation matrix $\boldsymbol{t}_{\mathrm{r}}=\left[\begin{array}{cc}\cos \delta_{\mathrm{GS}} & \sin \delta_{\mathrm{GS}} \\ -\sin \delta_{\mathrm{GS}} & \cos \delta_{\mathrm{GS}}\end{array}\right]$, $\boldsymbol{W}$ - vector of currents or voltages of the armature in different coordinate systems, $\delta_{\mathrm{GS}}=\delta-\delta_{\mathrm{S}}, \delta_{S}-$ position angle of the coordinate system $\mathrm{D}, \mathrm{Q}$ of the power system.

A model of the complete power system can be created by combining models of all generating units and taking into account the voltage and current equation of the reduced power network:

$$
\boldsymbol{I}_{\mathrm{WM}}=\boldsymbol{Y}_{\mathrm{sr}} \boldsymbol{U}_{\mathrm{WM}}
$$

where $\boldsymbol{I}_{\mathrm{WM}}, \boldsymbol{U}_{\mathrm{WM}}$ - vectors of the armature currents and voltages of all generating units in D, Q axis, $\boldsymbol{Y}_{\mathrm{sr}}-$ admittance matrix representing the equivalent power network which includes only generating nodes connected by means of artificial branches.

\section{Exemplary computations}

Exemplary optimisation computations were carried out for a 7-machine CIGRE power system shown in Fig. 3. It was assumed that all synchronous generators were represented by the GENROU turbogenerator model $[14,15]$, excitation systems - by the model of the national (Polish) static excitation systems [12, 13] and turbines - by the IEEEG1 steam turbine model [12-15]. Moreover, it was assumed that the PSS3B power system stabilizer was installed in all generation units [12, 13, 15] (Fig. 4).

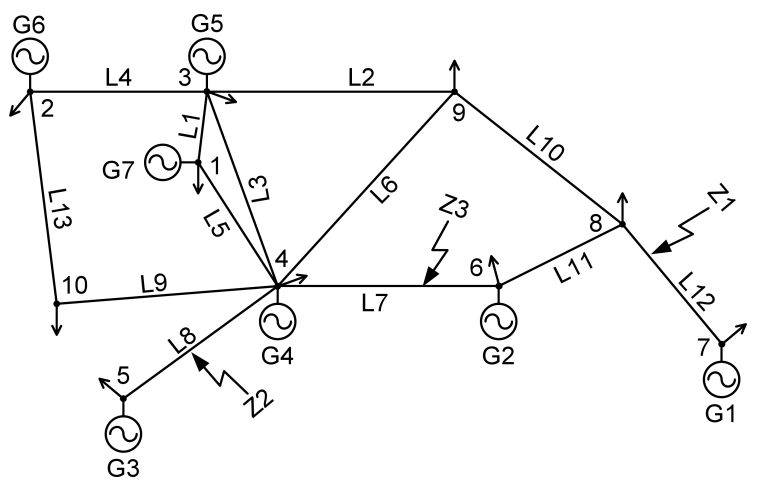

Fig. 3. CIGRE power system

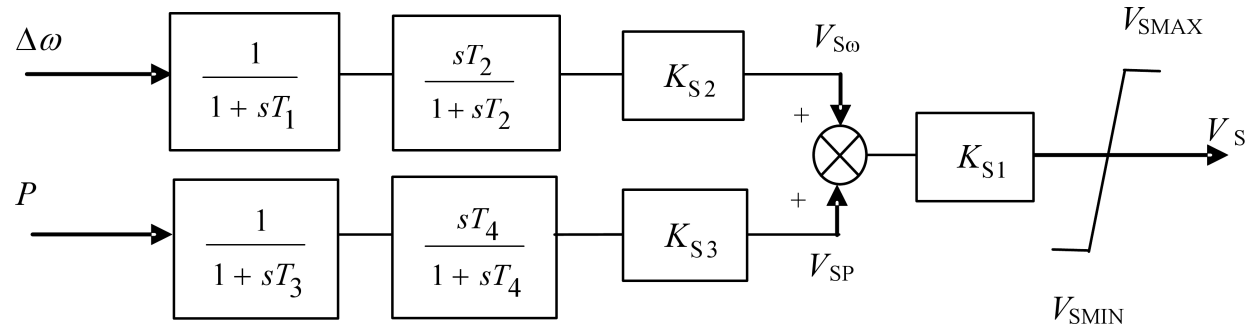

Fig. 4. The structural diagram of the dual-input power system stabilizer PSS3B 
When carrying out calculations, there was assumed that the time constants of particular PSSs had values from Table 1 (related to the results of single criterion parameter optimisation of those stabilizers in one-machine systems [12]). The parameters: $K_{S 1}=1, V_{S M A X}=0.2, V_{S M I N}=-0.066$ were assumed to be constant. For all cases of the Pareto optimisation performed it was assumed that the gain coefficients $K_{S 2}$ and $K_{S 3}$ of all PSSs were changed. Due to it, the dimension of the control vector was $\boldsymbol{X}: l=14$ (two gain coefficients changed in seven generating units).

Table 1

Time constants of PSS3B power system stabilizers

\begin{tabular}{ccccc}
\hline \hline \multirow{2}{*}{ Generating unit } & $T_{1}$ & $T_{2}$ & $T_{3}$ & $T_{4}$ \\
\cline { 2 - 5 } & $\mathrm{s}$ & $\mathrm{s}$ & $\mathrm{s}$ & $\mathrm{s}$ \\
\hline $\mathrm{G} 1$ & 0.01 & 5.0 & 0.052 & 5.0 \\
\hline $\mathrm{G} 2$ & 0.010 & 5.0 & 0.047 & 5.0 \\
\hline $\mathrm{G} 3$ & 0.100 & 4.99 & 0.036 & 0.101 \\
\hline $\mathrm{G} 4$ & 0.010 & 5.0 & 0.010 & 5.0 \\
\hline $\mathrm{G} 5$ & 0.100 & 5.0 & 0.071 & 5.0 \\
\hline $\mathrm{G} 6$ & 0.100 & 5.0 & 0.071 & 5.0 \\
\hline $\mathrm{G} 7$ & 0.054 & 5.0 & 0.100 & 5.0 \\
\hline
\end{tabular}

In the investigations presented there was performed the Pareto optimisation for different forms of the objective function of three component criteria.

At the first stage of calculations only one disturbance $\mathrm{Z1}$ of Fig. 3 in the form of a symmetrical short-circuit in line L12 $(110 \mathrm{kV})$ of duration time $t_{z}=0.25 \mathrm{~s}$ was taken into account. During the Pareto optimisation there were minimised the deviations of: the instantaneous power, the angular speed and the terminal voltage in the first and seventh generating unit. The form of the objective function for the generating unit G1 is given by the relationship (5), and for the generating unit G7 - by the formula (6):

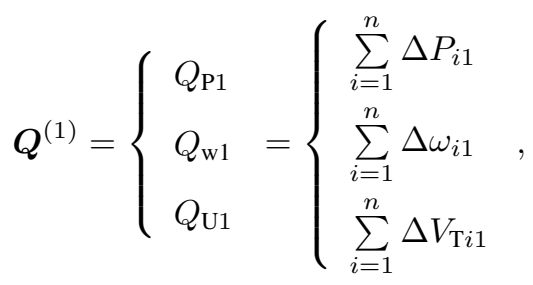

a)

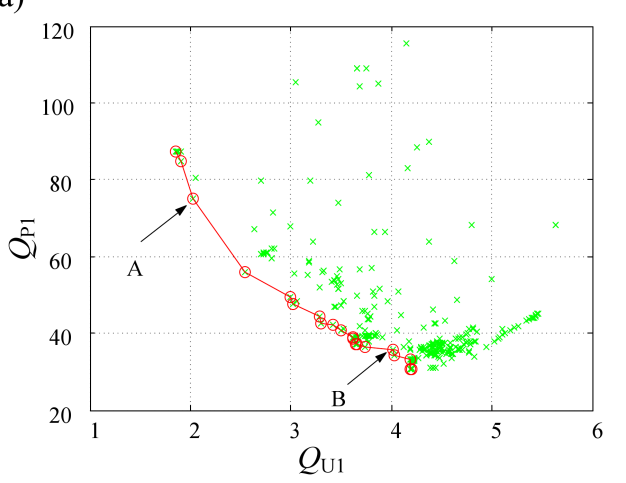

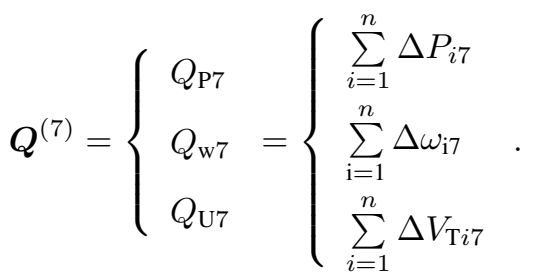

For both objective functions analysed there were assumed the following parameters of the genetic algorithm (at the first stage of the hybrid algorithm): population of 16 individuals, crossover probability equal to 0.9 , mutation probability equal to 0.05 , tournament group of 4 individuals. The genetic algorithm was stopped after 40 generations, and the final result was determined by verifying the condition (1) for all individuals in all generations. At the second stage of calculations the Newton constraint gradient algorithm was used.

As a result of optimisation there were determined two compromise sets (Pareto optimal sets) $\boldsymbol{\Lambda}$ presented in Figs. 5 and 6 . Moreover, in these figures there are located the points determined during the Pareto optimisation process as well as selected points (A, B, C and D) belonging to the compromise sets. Figs. 7 and 8 show selected waveforms in the network relative units (reference power $S_{\text {ref }}=100 \mathrm{MW}$ ) of the deviation of the angular speed, the terminal voltage and the instantaneous power in the first and seventh generating unit for a symmetrical short-circuit $\mathrm{Z} 1$ in the transmission line L12. Those waveforms concern the selected points of the compromise set, respectively: A, B for the generating unit G1 and C, D for the generating unit G7. The concrete values of the PSS gain coefficients in all generating units given in Table 2 correspond to the points $\mathrm{A}, \mathrm{B}, \mathrm{C}$ and $\mathrm{D}$ belonging to the compromise sets.

It is evident from Figs. 5 and 7 that for the point B of the compromise set the damping of the angular speed (also the instantaneous power) swings of the generator working in the generating unit G1 is better (comparing to the point A). Whereas, for the point $\mathrm{A}$ there are smaller deviations of the terminal voltage in the generating unit G1. It follows from Figs. 6 and 8 that for the point $\mathrm{D}$ of the compromise set the damping of the instantaneous power (also the angular speed) swings is a little better, and for the point $\mathrm{C}$ the deviations of the terminal voltage of the generator working in the generating unit G7 are significantly smaller.

b)

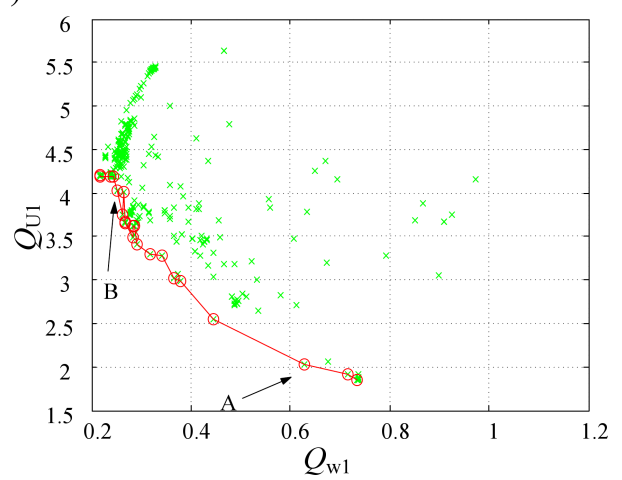

Fig. 5. Set of optimal Pareto solutions for short-circuit Z1 in line L12 when optimising criterion (5) 
a)

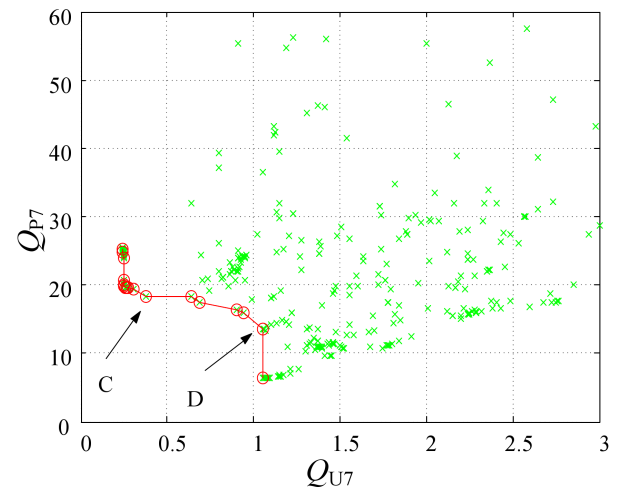

b)

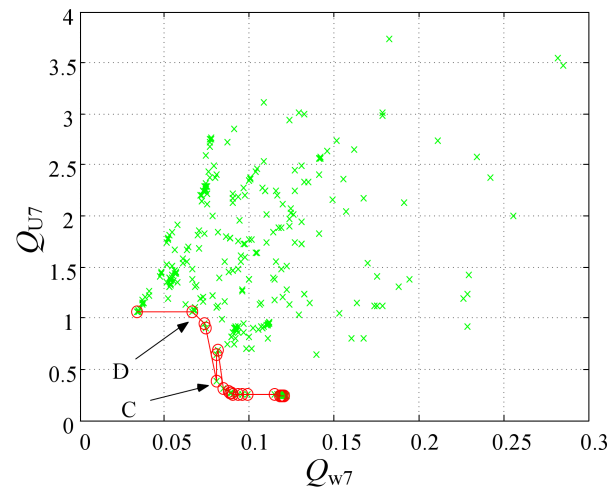

Fig. 6. Set of optimal Pareto solutions for short-circuit Z1 in line L12 when optimising criterion (6)

a)

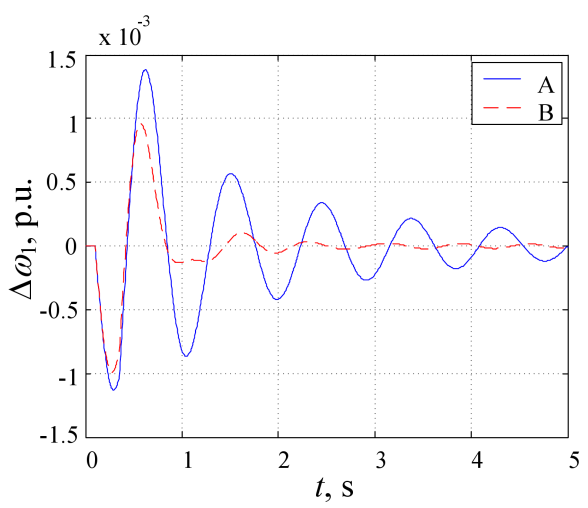

b)

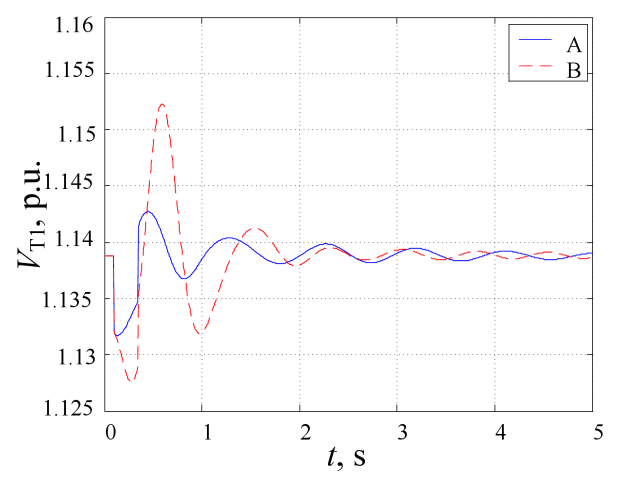

Fig. 7. Transients (per network units) of angular speed deviation (a) and terminal voltage (b) at node G1- disturbance Z1 for A and B points from the compromise set of Fig. 5

a)

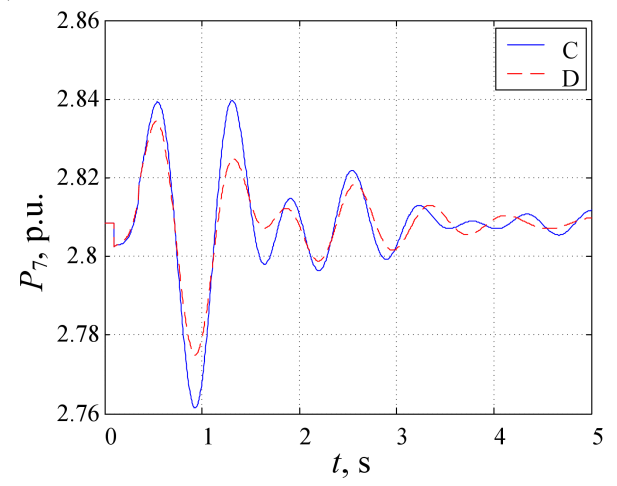

b)

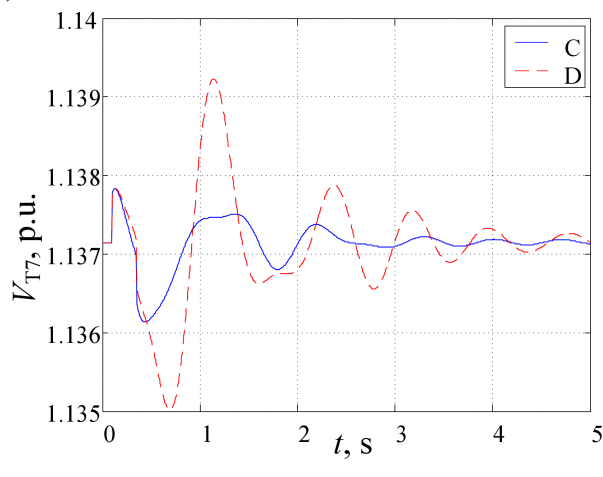

Fig. 8. Transients (per network units) of real power (a) and terminal voltage (b) at node G7- disturbance Z1 for C and D points from the compromise set of Fig. 6

Table 2

Parameters of PSS3B power system stabilizers corresponding to the points A, B, C and D from the compromise sets

\begin{tabular}{ccccccccc}
\hline \hline \multirow{2}{*}{ Generating unit } & \multicolumn{2}{c}{ Point A } & \multicolumn{2}{c}{ Point B } & \multicolumn{2}{c}{ Point C } & \multicolumn{2}{c}{ Point D } \\
\cline { 2 - 9 } & $K_{S 2}$ & $K_{S 3}$ & $K_{S 2}$ & $K_{S 3}$ & $K_{S 2}$ & $K_{S 3}$ & $K_{S 2}$ & $K_{S 3}$ \\
\hline G1 & 17.84 & 0.096 & 2.857 & 0.412 & 5.545 & 0.490 & 2.857 & 0.468 \\
\hline G2 & 12.86 & 0.396 & 19.05 & 0.460 & 13.26 & 0.460 & 14.92 & 0.294 \\
\hline G3 & 3.754 & 0.498 & 14.60 & 0.198 & 3.999 & 0.164 & 0.635 & 0.119 \\
\hline G4 & 16.13 & 0.425 & 20.00 & 0.373 & 6.860 & 0.374 & 2.222 & 0.333 \\
\hline G5 & 19.00 & 0.497 & 14.92 & 0.198 & 0.429 & 0.187 & 8.254 & 0.365 \\
\hline G6 & 12.61 & 0.489 & 3.175 & 0.183 & 15.39 & 0.472 & 5.397 & 0.095 \\
\hline G7 & 16.42 & 0.459 & 15.24 & 0.484 & 3.878 & 0.061 & 5.079 & 0.286 \\
\hline
\end{tabular}


a)

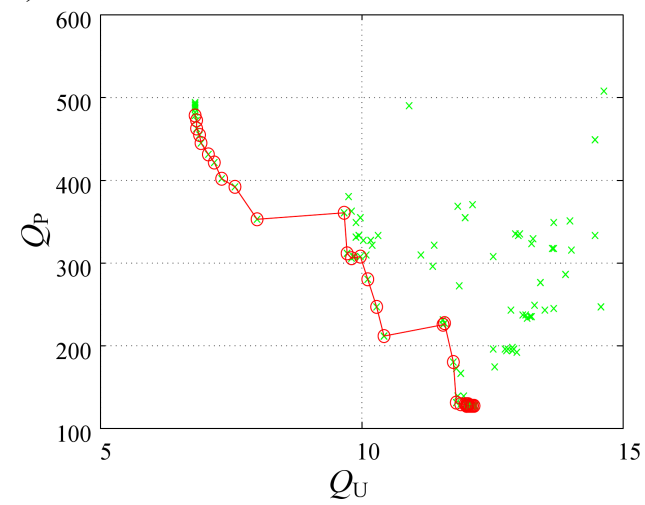

b)

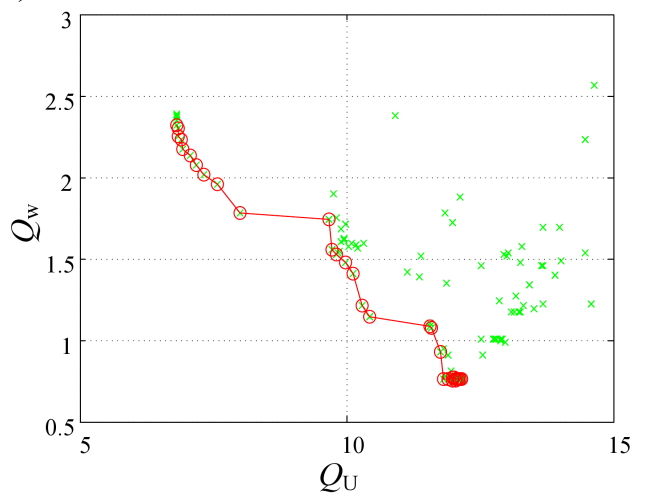

Fig. 9. Set of optimal Pareto solutions for short-circuit Z2 in line L8 when optimising criterion (7)

In next investigations there was performed the Pareto parameter optimisation of the PSSs when minimising at the same time the deviation of the instantaneous power, the angular speed and the terminal voltage in all generating units for three different disturbances Z1, Z2, Z3 (transient short-circuits in the transmission line L12, L8 and L7, respectively) of the analysed 7-machine PPS CIGRE. The objective function in this case is given by the relationship:

$$
\boldsymbol{Q}^{=}\left\{\begin{array}{l}
Q_{\mathrm{P}} \\
Q_{\mathrm{w}} \\
Q_{\mathrm{U}}
\end{array}=\left\{\begin{array}{c}
\sum_{j=1}^{7} \sum_{i=1}^{n} w_{\mathrm{p} j} \Delta P_{i j} \\
\sum_{j=1}^{7} \sum_{i=1}^{n} w_{\mathrm{p} j} \Delta \omega_{i j} \\
\sum_{j=1}^{7} \sum_{i=1}^{n} w_{\mathrm{p} j} \Delta V_{\mathrm{T} i j}
\end{array}\right.\right.
$$

where $w_{p j}$ - weighing coefficients are determined according to the relationship:

$$
w_{\mathrm{p} j}=\frac{n_{j} S_{\mathrm{N} j}}{S_{\max }},
$$

$n_{j}$ - number of generators working in the $j$-th generating unit, $S_{N j}$ - rated apparent power of a single synchronous generator working in the $j$-th generating unit, $S_{\max }$ - rated apparent power of a generating unit which generates the maximum apparent power.

Exemplary sets of the Pareto optimal solutions for the short-circuit $\mathrm{Z} 2$ in the line L8 are shown in Fig. 9.

\section{Compromise set deformation}

The important problem of the optimisation process is possibility of performing the assessment of the obtained solution from the point of view of a disturbing factor. The assessment of the solution and probable comparison with other solutions is usually made by analysis of the influence of the disturbing factor under consideration on the value of the factor (objective function) assumed for optimisation. Such assessment can be made both for the single criterion and Pareto optimisation. In case of the Pareto optimisation the disturbance can influence selected or all quality criteria. A concept of the compromise set "deformation" under the influence of the investigated dis- turbance has been introduced [2]. The compromise set deformation is a measure of the influence of a disturbance on the control quality in the investigated system. In the general case, the disturbance can influence the all criteria analysed.

For PPS analysis one can assume the quality criteria to be described by, for instance, dependencies (5)-(7), while a disturbing factor can be, for instance, change of the PS operating condition or the uncertainty of the system element mathematical models. In publication [7] there are presented investigations concerning the influence of changes of the mathematical model parameters of the elements of generating units working in PS on the quality criteria analysed. There was determined the factor of relative change of the PS model parameter value (from the given range). From the presented investigation results it follows that there exist such optimal solutions (values of PSS parameters) for which the PS analysed is more robust to changes of the system model parameters. PSSs of such parameters, which can be conventionally called "robust stabilizers", damp electromechanical swings well without causing deterioration of the control waveforms of generator voltages for different parameters of the analysed power system model [7].

\section{Concluding remarks}

There has been presented the method for determining the sets of Pareto optimal solutions (compromise sets) - parameter values of PSSs working in a multi-machine PS - when optimising different multidimensional criteria. Those criteria have been determined for concrete disturbances when taking into account transient waveforms in one, selected generating unit (formulas 5 and 6) or in all units of the analysed system (formula 7).

On the basis of the analyses performed, the following general conclusions can be drawn:

- The used Pareto optimisation of PSS parameters enables efficient damping of electromechanical swings without significant worsening the terminal voltage regulation waveforms of generating units in the PS analysed.

- Application of multi-criteria methods allows taking into account in the PSS parameter optimisation process many 
(in the presented investigations 3 criteria), sometimes contradictory requirements (criteria) without loss of ability to achieve an optimal solution.

- The hybrid algorithm used makes it possible to fast determine optimal parameters of many PSSs simultaneously.

- Criteria associated with electromechanical swings, that is disturbance waveforms of the instantaneous power and angular speed of particular generators have similar properties. In the system in which there occur large damping of the instantaneous power, the angular speed of generators is also damped well.

- Criteria associated with deviations of the generator terminal voltage are usually contradictory to those associated with electromechanical swings. If electromechanical swings are well damped, there usually occur significant deviations of the generator terminal voltage, and the contrary.

- Despite the fact that the criteria associated with the deviations of the generating unit voltage are usually contradictory to those associated with electromechanical swings, it is possible to find in the compromise sets the ranges, e.g. between points $\mathrm{C}$ and $\mathrm{D}$ in Fig. 6, for which there occur large changes of the criterion $Q_{U 7}$ value (increase in the value from point $\mathrm{C}$ to $\mathrm{D}$ ) at only small changes (decrease in the values) of the criteria $Q_{P 7}$ and $Q_{w 7}$. In other words, one can find controls (values of PSS parameters) for which the regulation waveforms of generator voltages are significantly improved at only insignificant worsening the damping of electromechanical swings.

- For the quality criteria taking into account disturbance waveforms in different generating units the compromise sets presented are less sharp. There occur some averaging of the influence of disturbance waveforms in different places of PS. In case of the Pareto optimisation, at the stage of calculations the disturbance waveforms of different quantities are not brought together in one, generalized quality factor.

- There is a possibility to select settings of PSSs in such a way that sensitivity of PS to changes of its parameters and configuration is decreased [7].

\section{REFERENCES}

[1] E. Bednarczuk, Parametric Problems of Multicriteria Optimisation, Academic Publishing House EXIT, Warszawa, 2005, (in Polish).
[2] A. Nocoń and S. Paszek, Polyoptimisation of Voltage Regulators of Generating Units with Synchronous Generators, Silesian University of Technology Publishing House, Gliwice, 2008, (in Polish).

[3] A. Nocoń and S. Paszek, "Synthesis of generator voltage regulator when applying polyoptimisation", Bull. Pol. Ac.: Tech. 55 (1), 43-48 (2007).

[4] Z. Bubnicki, Control Theory and Algorithms, PWN, Warszawa, 2002, (in Polish).

[5] M. Peschel and C. Riedel, Polyoptimierung - eine Entscheidungshilfe für ingenieurtechnische Kompromislösungen, VEB Verlag Technik, Berlin, 1976.

[6] S. Paszek, "Optimisation of the power system stabilizers in a power system", Scientific Notesbooks of Silesian University of Technology Publishing House, Electrics 161, CD-ROM (1998), (in Polish).

[7] A. Nocoń and S. Paszek, "Analysis of power system stabilizer Pareto optimization when taking into account the uncertainty of power system mathematical model parameters", Archives of Electrotechnics 4, CD-ROM (2011).

[8] K. Hongesombut, S. Dechanupaprittha, Y. Mitani, and I. Ngamroo, "Robust power system stabilizer tuning based on multi objective design using hierarchical and parallel micro genetic algorithm", 15th PSCC 28, CD-ROM (2005).

[9] M. Khaleghi, M. Mohammadian, M. Khorasaninejad, and M.M. Farsangi, "Design of two power system stabilizers coordinately based on strength Pareto evolutionary algorithm", IEEE ICIEA 1, 1527-1531 (2009).

[10] P. X. Zhang, Y. J. Cao, and S. J. Cheng, "Coordination of multiple PSSs using multi-objective genetic algorithm", Proc. 5th World Congress on Intelligent Control and Automation 1, 5040-5044 (2004).

[11] A. Andreoiu and K. Bhattacharya, "Genetic algorithm based tuning of PID power system stabilizers", 14th PSCC, Session 14, CD-ROM (2002).

[12] S. Paszek and A. Pawłowski, "Application of power system stabilizers PSS3B to electromechanical swing damping in multimachine power system", Acta Technica CSAV, Academy of Sciences of the Czech Republik 53 (2), 159-171 (2008).

[13] S. Berhausen and S. Paszek, "Parameter estimation of the model of a synchronous generator working in multi-machine power system”, Electrotechnical Review 8, 192-197 (2011), (in Polish).

[14] A. Boboń, S. Paszek, P. Pruski, T. Kraszewski, and M. Bojars$\mathrm{ka}$, "Computer-aided determining of parameters of generating unit models basing on measurement tests", Electrotechnical Review 87 (5), 17-21 (2011), (in Polish).

[15] Power Technologies, a Division of S\&W Consultants Inc., Program PSS/E Application Guide. 